\title{
A Portable Ankle-Foot Rehabilitation Orthosis Powered by Electric Motor
}

\author{
Yang Bai ${ }^{*}, 1$, Xueshan Gao ${ }^{1}$, Jun Zhao ${ }^{2}$, Fei $\mathrm{Jin}^{3}$, Fuquan Dai ${ }^{1}$ and Yunqi Lv ${ }^{1}$ \\ ${ }^{1}$ School of Mechatronical Engineering, Beijing Institute of Technology, Beijing, 100081, China \\ ${ }^{2}$ Department of Neurological Rehabilitation, China Rehabilitation Research Center, Beijing, 100068, China \\ ${ }^{3}$ Rehabilitation Engineering Research Institute, China Rehabilitation Research Center, Beijing, 100068, China
}

\begin{abstract}
Powered ankle-foot orthosis can not only prevent foot-drop and assist patients' walking but also improve the ankle joint movement for patients with dysfunction caused by the various injuries and nervous system diseases. Common ankle rehabilitation devices limit the ankle injury patients' rehabilitation training within fixed places, so a portable powered ankle-foot orthosis is presented in this paper to enable the patients to continue their work normally with the treatment. The orthosis employs electric motor drive mode to provide ankle dorsiflexion and plantar flexion assistance during patient's walking. First, the ankle-foot dynamics model is established and the gait is analyzed for the powered ankle-foot orthosis system. Then, a new mechanical structure including wearing parts, analogous ankle joint and transmission is described. For the small installation space between the instep and the knee, the compact transmission mechanism has been given more attention and the finite element method is adopted to optimize the key structure after the force analysis. In addition, the closed-loop control system is chosen for the orthosis position and speed control. At last, wearing and movement experiments on the prototype are carried out, which validates the stability and rationality of the structure design and the effectiveness of the motion control. It has great significance in promoting patient's rehabilitation to help them return to the society.
\end{abstract}

Keywords: Ankle-foot, ankle rehabilitation, dorsiflexion, plantar flexion, powered orthosis.

\section{INTRODUCTION}

The technologies of rehabilitation robots are mainly promoted by the increasing demands from vulnerable people, such as the elderly and the disabled. Ankle injuries are common in two kinds of patients: one type is the patients with fracture and sprain caused by traffic accidents or natural disasters, and the other type is the patients with nervous system disorders like hemiplegia resulting from brain paralysis and stroke. From World Stroke Organization's latest report, about 17 percent of people encounter a stroke in their lifetime. Every year, 16 million people have a stroke out of whicht 6 million die of the disease [1]. This serious ankle movement dysfunction affects the weight-bearing ability of patients' lower limbs and their social participation, which eventually increases the burden of their families and society. Moreover, in the recovery stage, if the ankle joint is not given effective and sufficient rehabilitation training, the reversible injury like drop-foot, varus and valgus may change to irreversible injury, even permanent disability [2]. Hence, preventing and treating ankle injuries is an important nursing work.

There are lots of therapeutic methods in the treatment of ankle injuries, including physiotherapy, electromyographic biofeedback therapy, surgical treatment and orthosis

*Address correspondence to this author at the School of Mechatronical Engineering, Beijing Institute of Technology, Beijing, 100081, China; Tel: 86-13581927345; E-mail: byang@bit.edu.cn treatment. Among these methods, orthosis as an advanced and effective method, has been widely used in rehabilitation training, from rigid ankle-foot orthosis (R-AFO) like simple passive bandages to dynamic ankle-foot orthosis (D-AFO) and powered ankle-foot orthosis (P-AFO) as adjustable orthoses. Ankle foot orthosis (AFO), with the structure covering foot and lower leg, prevents joint deformation and muscular atrophy by controlling and limiting ankle motion. Furthermore, as a safe and reliable device, AFO can take the place of therapists for patients' rehabilitation training for the therapeutic purpose of deformation prevention and correction, and motion control.

$\mathrm{R}-\mathrm{AFO}$, a traditional AFO, which is fixed and linked by hard splint or is drawn to the model with thermoplastic mold, has the advantages of low price and simple structure. But once formed, the R-AFO cannot be resized, so it keeps the ankle's position fixed [3]. This obvious disadvantage hampers its application and may produce unwanted side effects. Compared with R-AFO, the D-AFO has an outstanding advantage that an adjustable angle joint connecting the foot and lower leg parts enables the ankle to plantar flex and dorsiflex to a certain extent. Although the DAFO is an improvement relative to R-AFO, it still cannot provide needed power actively. The ankle injury patients fatigue easily, since they consume more energy than normal persons, that is, their bodies' metabolism is accelerated [4, 5]. It has been found [6] that increasing assisted moment at the ankle could lower the metabolic rate.

Therefore, P-AFO is presented as a typical assistive robot. In the past, the majority of global researchers in the 
field of powered lower limb rehabilitation devices focused on the hip, thigh, knee and lower leg orthoses and they usually use these powers to make up for the loss of ankle power. But nowadays, the economic and social value has promoted researchers and entrepreneurs to explore the viability of the P-AFO for ankle rehabilitation. Moreover, the advances in hydraulic system, pneumatic muscle, elastic energy storage and electrical motor technologies provide various methods to produce the power for ankle joint. Because most of the ankle motion occurs in the sagittal plane which outweighs the motion in the transverse and frontal planes [7-9], P-AFOs' function mainly focuses on the dorsiflexion and plantar flexion rehabilitation training and assistance. A self-controlled P-AFO using a bidirectional pneumatic rotary actuator was presented to generate the needed power $[10,11]$. An actuated cam-lock mechanism was designed to accomplish the ankle motion by pressurizing the pump located in the sole of the foot. The P-AFO does not need the external energy source to provide the power, but it cannot control the gait and torque during the entire period of patients' walking and it is not suitable for the patients with motion deformation. Characterized by light weight and high power, pneumatic muscle has been applied gradually in developing the P-AFO. As a typical representative, a kind of $\mathrm{P}-\mathrm{AFO}$ s with a carbon fiber and polypropylene using artificial pneumatic muscles was described by Ferris et al. [12-15]. They are able to provide plantar flexion and dorsiflexion respectively in two orthoses or together in the same orthosis depending on the pneumatic muscles' positioning, in the front or rear, or both. In addition, combining the characteristics of electric and hydraulic systems, an electrohydraulic AFO was proposed with two cylinders connected by hoses and controlled by an electric motor. It could provide ankle pronation-supination motion [16]. For these P-AFOs using external pneumatic and hydraumatic power supplies, the application is limited to the laboratory or some given space due to the large size and heavy weight power sources. For evaluation of the portability, the P-AFOs driven by electrical motors with a series elastic actuator were developed by Herr et al. [3, 17] from Massachusetts Institute of Technology and Ward et al. [18] at Arizona State University. They provide plantardorsiflexion torque by the adjustable impedance. Both the two P-AFOs are the rear-AFOs that most of the appeared PAFOs belong to. Compared with front-AFO, although the rear-AFO has sufficient space to install the powerplant, the collision occurs easily in extra space and it is inconvenient for observation by the user.

By the comprehensive comparison of various P-AFOs in driving modes, actuators and power transmission ways, a lightweight, portable and front P-AFO providing ankle dorsiflexion and plantar flexion assistance for ankle rehabilitation with motor-drive mode is presented in this paper to overcome the weakness of the common ankle rehabilitation devices limiting the patients within fixed places and to enable the patients to continue their normal work with it. Equipping AFO has great realistic significance for patient's rehabilitation to help them return to society and reduce the burden of society. A major challenge of developing the $\mathrm{P}-\mathrm{AFO}$ is the design of transmission mechanism to ensure the compactness and portability due to the limit of small space between the instep and the knee in the front P-AFO. The paper is organized as follows. In the following Section 2, the dynamics and gait are analyzed for motion planning. Then the design concept, characteristics, force analysis and control system of the P-AFO are described in Section 3.1 to Section 3.3. In Section 3.4, the P-AFO prototype is displayed and worn by two subjects. To verify the performance of the P-AFO, preliminary experiments on the prototype were conducted in no-load and walking-aid modes as presented in Section 4. Finally, Section 5 generalizes the conclusions.

\section{DYNAMICS AND GAIT ANALYSIS}

\subsection{Dynamics Model}

The kinematics and dynamics information related to human walking has important significance in the evaluation of rehabilitation effects and monitoring of surgical efficiency. The ankle plantar flexion and dorsiflexion in the sagittal plane is focused on in this paper. The ankle-foot model and the curves of angle variations of ankle joint plantar-dorsiflexion have a vital influence on the transmission mode and motor selection, and motor position and speed control. So the dynamics and gait analysis are a necessary foundation for orthosis research.

To control the motor and gain better rehabilitation effects, the force and torque around ankle should be analyzed. When people walk normally on the ground, foot is subjected to combined ground reaction force, ankle reaction force and muscle forces. There is tibialis anterior muscle in front of the ankle joint and crus triceps at the back. At singlestance phase, the gravity torque causes the shank to lean back, and then the tibialis anterior muscle force increases and the crus triceps force is very small, so the crus triceps can be neglected; at terminal single-stance period, tibialis anterior muscle contracts to resist the gravity torque causing the ankle to dorsiflex and the body to lean forward, therefore, the crus triceps force increases and tibialis anterior muscle force is very small. Muscle forces (mainly the dorsiflexor muscle force and plantar flexor muscle force) around the ankle joint can be modeled as simple springs. So it is assumed that there is only joint stiffness contributed by the dorsiflexor muscle force when the ankle dorsiflexes, and the spring standing for plantar flexor muscle force only exists when the ankle plantar flexes $[19,20]$. The dynamics analysis of the ankle-foot model as the ankle dorsiflexes is shown in Fig. (1). The forces are decomposed in the sagittal plane. A is the ankle center location, COM is the center of mass of the foot and COP is the center of pressure.

On the basis of Newton's second law, the resultant force on ankle-foot is expressed as [20,21]:

$\sum \mathbf{F}=\mathbf{F}_{G R F}+\mathbf{F}_{A}+m_{\text {foot }} \mathbf{g}+\mathbf{F}_{k}=m_{\text {foot }} \mathbf{a}_{\text {foot }}$

where $\boldsymbol{F}_{G R F}$ is ground reaction force, $\boldsymbol{F}_{A}$ is ankle reaction force, $m_{\text {foot }}$ is mass of the foot, $g$ is acceleration of gravity, $\boldsymbol{F}_{k}$ is elastic force applied by the spring and $\boldsymbol{a}_{f o o t}$ is foot linear acceleration vector.

Equation (1) can be rewritten in the sagittal plane as: 


$$
\begin{aligned}
& {\left[\begin{array}{c}
-F_{G R F x} \\
F_{G R F z}
\end{array}\right]+\left[\begin{array}{c}
F_{A x} \\
-F_{A z}
\end{array}\right]-\left[\begin{array}{l}
0 \\
1
\end{array}\right] m_{\text {foot }} g+\left[\begin{array}{c}
\sin \theta \\
\cos \theta
\end{array}\right] k r_{3}|\theta|} \\
& =m_{\text {foot }}\left[\begin{array}{c}
\ddot{x}_{\text {foot }} \\
\ddot{z}_{\text {foot }}
\end{array}\right]
\end{aligned}
$$

where $F_{G R F x} / F_{G R F z}$ is horizontal/vertical ground reaction force, $\ddot{x}_{\text {foot }} / \ddot{z}_{\text {foot }}$ is foot horizontal/vertical linear acceleration, $F_{A x} / F_{A z}$ is horizontal/vertical ankle reaction force, $k$ is spring constant, $r_{3}$ is length between the spring and $\mathrm{A}$ and $\theta$ is angle of the ankle joint.

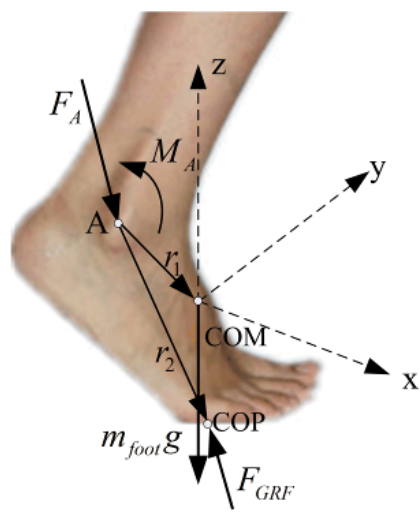

(a)

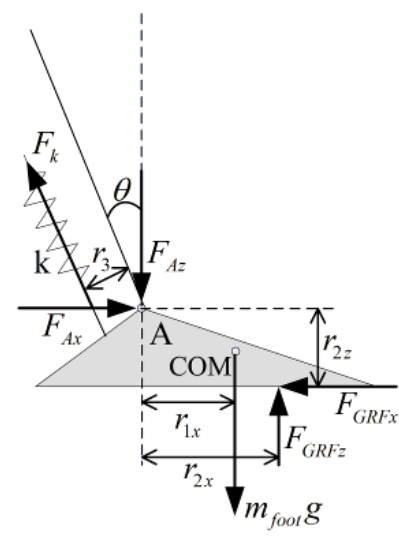

(b)
Fig. (1). Ankle-foot model.

When the foot is in static equilibrium, that is, foot linear acceleration equals zero, ground reaction force is computed as:

$$
\left\{\begin{array}{l}
F_{G R F x}=F_{A x}+\sin \theta k r_{3}|\theta| \\
F_{G R F z}=F_{A z}+m_{\text {foot }} g-\cos \theta k r_{3}|\theta|
\end{array}\right.
$$

The resultant moment of forces acting on the ankle is written as:

$$
\begin{aligned}
\sum \boldsymbol{M} & =\mathbf{M}_{A}+\mathbf{r}_{1} \times m_{\text {foot }} \mathbf{g}+\mathbf{r}_{2} \times \mathbf{F}_{G R F}+\mathbf{M}_{k} \\
& =I \boldsymbol{\alpha}_{\text {foot }}
\end{aligned}
$$

where $\boldsymbol{M}_{A}$ is ankle reaction moment, $\boldsymbol{\alpha}_{\text {foot }}$ is foot angular acceleration rotating around $\mathrm{A}, \boldsymbol{r}_{1} / \boldsymbol{r}_{2}$ is position vector of $\mathrm{COM} / \mathrm{COP}$ relative to A and $\boldsymbol{M}_{k}$ is spring reaction moment.

The moment equation in the sagittal plane can be given as:

$$
M_{A}-r_{1 x} m_{\text {foot }} g+r_{2 x} F_{G R F z}-r_{2 x} F_{G R F x}+k r_{3}^{2} \theta=I|\ddot{\theta}|
$$

where $I$ is moment of inertia, $\ddot{\theta}$ is angular acceleration of ankle centre location, $r_{1 x}$ is horizontal distance from $\mathrm{A}$ to $\mathrm{COM}, r_{3}$ is length between the spring and $\mathrm{A}, r_{2 x} / r_{2 z}$ is horizontal/vertical distance from A to COP.

When the foot is in static equilibrium, combining equation (3) and equation (5), the ankle reaction moment about the ankle of the ankle joint can be calculated as:

$$
\begin{aligned}
M_{A}= & \left(r_{1 x}-r_{2 x}\right) m_{f o o t} g-r_{2 x} F_{G R F z}+r_{2 z} F_{G R F x} \\
& +\left(r_{2 x} \cos \theta+r_{2 x} \sin \theta\right) k r_{3}|\theta|-k r_{3}^{2} \theta+I|\ddot{\theta}|
\end{aligned}
$$

The ankle power can be obtained:

$P=M_{A} \cdot \dot{\theta}$

where $\dot{\theta}$ is Angular velocity of the ankle joint.

\subsection{Gait Analysis}

Walking is the basis of human normal activity while gait can reflect human health condition and pathological feature to some extent. Several gait characteristics are included in the gait cycle that is an important basis for motor control. The process from one heel striking the ground to the same one striking again is shown in Fig. (2). According to foot's position, the gait cycle is divided into stance phase and swing phase: stance phase is when the foot contacts the ground and bears human's weight, taking up about 60 percent of gait cycle; swing phase occurs between toe off and heel striking, accounting for 40 percent of gait cycle.

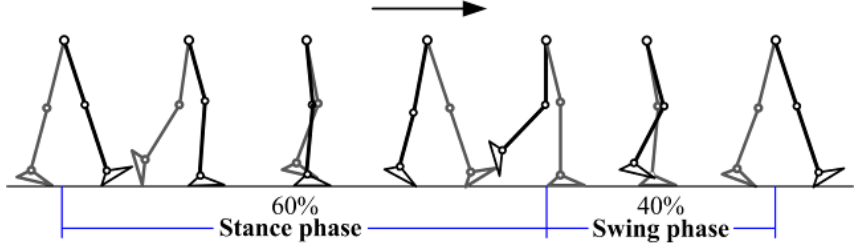

Fig. (2). Gait cycle.

The human averaged walking cadence is from 111 steps/minute to $122 \mathrm{steps} / \mathrm{min}$ with the people aging from 20 to 65 years [22]. Using cubic interpolation and fragmented curve-fitting method, the ankle plantar-dorsiflexion motion curve is obtained based on the reference [21-23] to control the motor motion as shown in Fig. (3). Seven key points in which the motor switches directions are chosen as the reference points in the ankle motion cycle. A negative value stands for plantar flexion and a positive value means dorsiflexion.

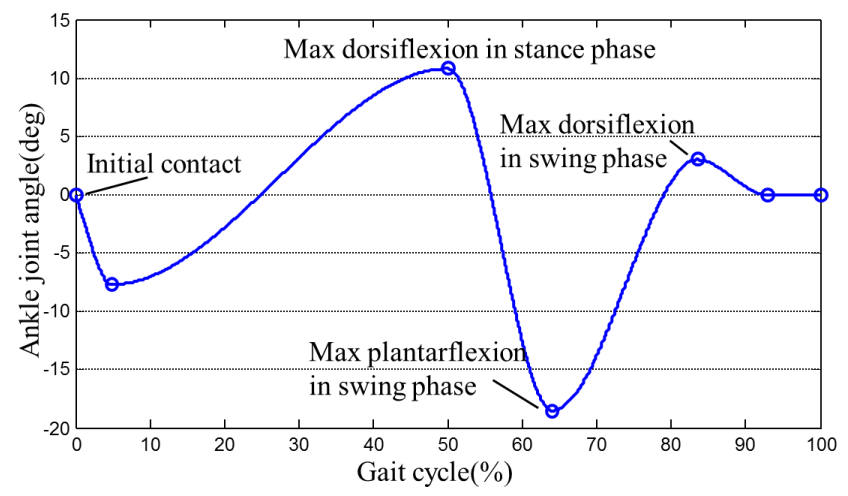

Fig. (3). Ankle joint control curve.

\section{AFO SYSTEM}

\subsection{Mechanical Structure}

The first step of developing the AFO system is the mechanical structure design, which directly influences the function realization of the whole system. There are some design principles in the design process: 1) the AFO's shape and size meet the demand of the body's physiological 


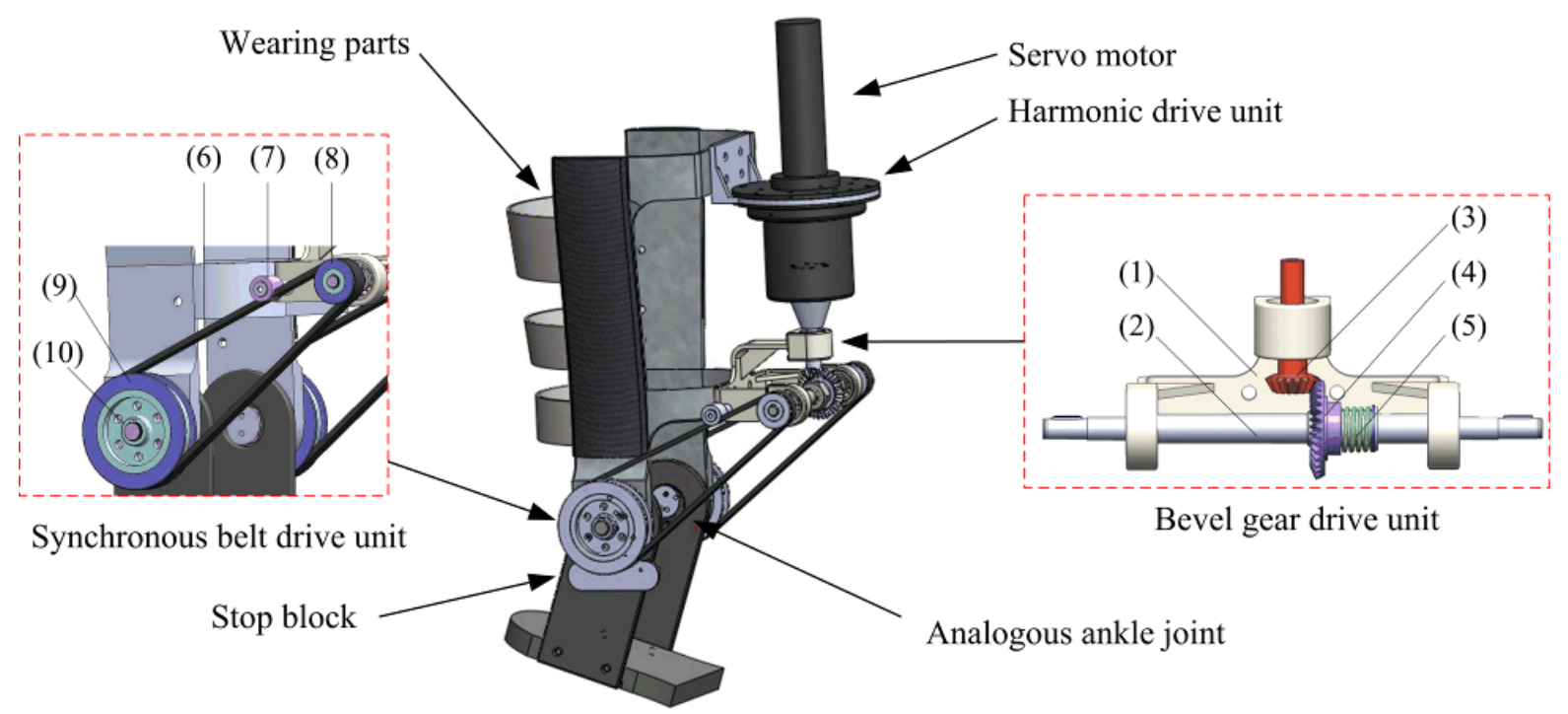

Fig. (4). 3D structure of the proposed P-AFO.

structure; 2) the range of AFO's ankle joint motion satisfies the angle changes of ankle plantar flexion and dorsiflexion during human's walking ; 3) under the premise of ensuring the mechanical strength, the AFO possesses the characteristics of portability, comfort and safety; 4) every part can connect/disconnect from other parts simply; 5) the power can be transmitted to the ankle joint steadily and accurately.

This P-AFO is a type of electromechanical integration equipment for assisted treatment of ankle injuries, which consists of the powertrain and wearing parts. The 3D structure design of the P-AFO is shown in Fig. (4).

\subsubsection{Powertrain}

The powertrain is composed of an actuator and transmission: the actuator is the elementary unit to provide energy for AFO movement; the transmission is responsible for transmitting mechanical energy to analogous ankle joint in a suitable ratio and generating motion torque and speed.

Servo motor, with the characteristics of high accuracy and mature control algorithms, is the kernel part of the system. Maxon's brushless DC motor EC-powermax 30 (rated power $200 \mathrm{~W}$, nominal voltage $24 \mathrm{~V}$ ) is chosen because of its small volume, light weight, high efficiency and good linearity.

In general, the powerplant is installed in the space between the knee and the foot, namely around the lower legs, because placed above the knee, it can affect the knee's flexion. Then if the powerplant is positioned close to the medial leg, the installing space is small and it can affect the other leg's movement; if the powerplant is mounted near the lateral leg, two different AFOs are needed for the leg and right ankle-foot rehabilitation, which decreases the universalism. By contrast, the front and rear AFOs avoid the above mentioned problems effectively. In addition, their symmetrical structure satisfies the rehabilitation requirements of both the left and right ankle with one AFO as well as reduces the weight and manufacture costs. In comparison with the front AFO, although the rear AFO has sufficient space to install the powerplant, the collision occurs easily in the extra space and it is inconvenient for the user to observe. Thus, compact structure of front AFO is proposed in this paper. So, the servo motor is placed in front of the lower leg and perpendicular to horizontal plane without affecting patients' walking.

After determining the actuator, the way of transmitting power needs to be considered due to the limit of the small space over the instep and the influence of ankle dorsiflexion, the movement of which occupies the space in front of the lower leg. Ultimately, the way of transmitting the power in both sides of the lower leg is chosen to generate the torque and speed for the analogous ankle joint.

The transmission composed of a harmonic drive, bevel gear and synchronous belt units not only makes the system's integration and installation easy, but also benefits maintenance and replacement in the event of part failure, which would be helpful in volume production.

- Harmonic drive unit: The harmonic drive XB1-50-80 (Beijing CTKM Harmonic Drive Co., Ltd.) with large reduction rate $(80: 1)$ and high strength bearing high transmission power is chosen.

- $\quad$ Bevel gear unit: Because the motor's rotating shaft is vertical while the ankle joint's rotating shaft is horizontal, the transmission should have the function of changing the movement direction.

In the selection of the direction-changing mechanism, the following mechanisms were considered in the design process:

1) Worm gearing has high transmission ratio, but the transmission efficiency is low, and the high speed of relative slip between gear teeth results in serious wear, which does not fit the long-duration working in high power.

2) Cardan shaft has complex and small angle variation range. Its transmission efficiency reduces with the angle increasing. 
3) Flexible shaft with compact structure is suitable for high speed and small torque.

In summary, for considering small space, large torque and other factors, bevel gear drive (Fig. 4) is adopted to change the transmission direction by $90^{\circ}$ while reducing the speed and increasing the torque. The simple compressed spring (Fig. 4) is effective for eliminating the gear backlash and then improving the transmission accuracy. In Fig. (4), (1) is bearing block, (5) is compressed spring, small bevel gear (3) connected with output shaft (2) of harmonic drive unit is the driving gear, and big bevel gear (4) is the driven gear.

Synchronous belt unit: The synchronous belt unit (Fig. 4) transmits torque from the bevel gear unit to the analogous ankle joint in both sides of the lower leg and is able to absorb impact. Its arc tooth structure and encapsulation in a small module size enhance the system's stability and lower the transmission noise.

For patients with ankle injury, the ligament, muscle, skeleton or nervus near ankle are sensitive to rotating motion range. The over-movement may cause patients' pain or secondary ankle injury, so the stop blocks (Fig. 4) are important for safety to prevent ankle injury from program running out and other situations resulting in the overmovement. In Fig. (4), (6) is 168XL-Arc tooth synchronous belt, (7) is tension pulley, (8) is small belt wheel with 12 teeth, (9) is big belt wheel with 36 teeth, and (10) is analogous ankle joint.

\subsubsection{Wearing Part}

For the patients' wearing convenience, the structure shape and size are similar to a human lower limb. An analogous ankle joint, connecting lower leg and foot wearing parts together, helps the AFO to complete plantar-dorsiflxion motion around the ankle joint. The foot wearing part is located at the heel, thus it does not affect the normal flexion of toes, and meanwhile, it decreases the AFO's weight.

\subsection{Force Analysis and Optimization}

Analysis and design software SolidWorks is used to select the most suitable dimensions and shape for the key structure. For example, the supporting mechanism which bears both internal forces from torque transmission and external forces from the load is needed to be optimized. A bearing block, connected with the synchronous belt and the end of transmission mechanism, experiences higher tension. Meanwhile, it is affected by the stress from the small bevel gear. So the stresses in the bearing block are analyzed by finite element method. Firstly, acquiring the forces of transmission system is necessary for the finite element analysis (Fig. 5).

In the synchronous belt unit (Fig. 5a), two big belt wheels offer the torque $T$ that the ankle injury patients need, that is, each one provides the torque $T_{2}=T / 2$.

The transmitted torque of small belt pulley can be given as: $T_{2}=T d_{1} / 2 d_{2}$.

where $d_{1} / d_{2}$ is reference diameter of small / big belt pulleys.

In ideal condition, supposing that initial tension is controlled properly, so the axial force of synchronous belt drive equal to the effective tension can be expressed as [24]:

$$
F_{Q}=F=F_{1}-F_{2}=\frac{P_{d}}{v}
$$

where $F_{1} / F_{2}$ is Tight/slack side tensile force, $P_{d}$ is the design power and $v=\frac{d_{1} P_{1}}{2 T_{1}}$ is Belt speed.

The axial force of synchronous belt drive can be rewritten as:

$$
F_{Q}=\frac{2 P_{d} T_{1}}{d_{1} P_{1}}
$$

where $P_{1}$ is the rated power.

In the gear drive unit (Fig. 5b), the radial forces $F_{r 3} / F_{r 4}$, axial forces $F_{a 3} / F_{a 4}$ and circumferential forces $F_{t 3} / F_{t 4}$ of driving and driven wheels are computed as [25]:

$F_{r 3}=F_{a 4}=\frac{2 T_{3}}{d_{3}} \tan \alpha \cos \delta_{3}$

$F_{r 4}=F_{a 3}=\frac{2 T_{3}}{d_{3}} \tan \alpha \sin \delta_{3}$

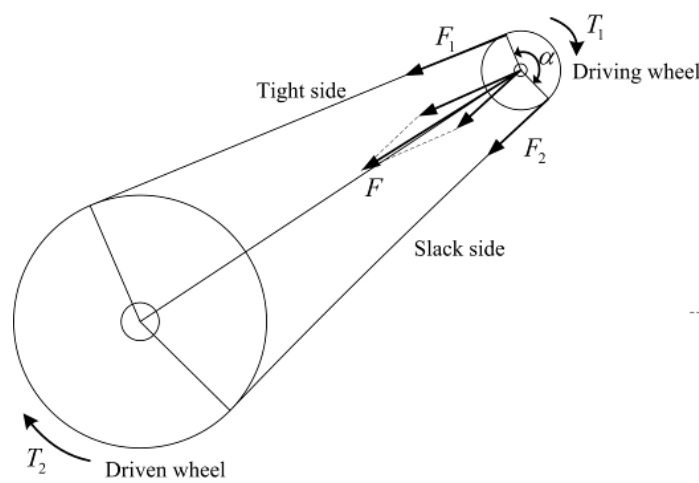

(a)

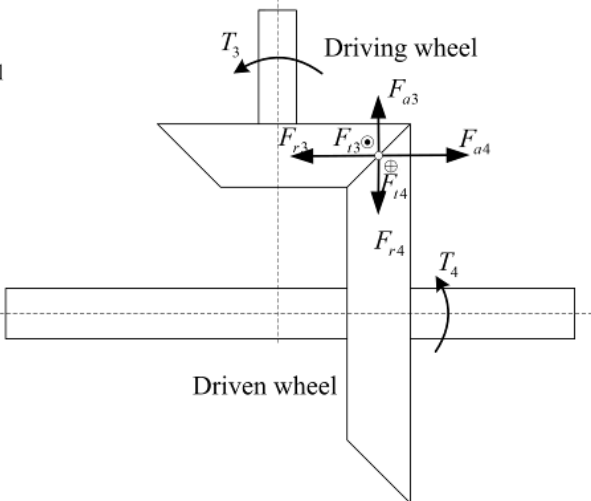

(b)

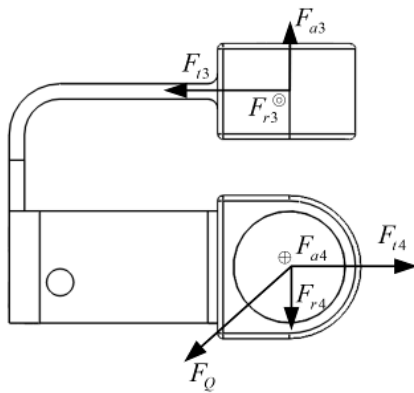

(c)

Fig. (5). Transmission system free body diagrams. 
$F_{t 3}=F_{t 4}=\frac{2 T_{3}}{d_{3}}$

where $T_{3}$ is the transmitted torque of small bevel gear, $d_{3}$ is reference diameter of small gear drive, $\alpha$ is pressure angle of Reference circle, and $\delta_{3}$ is reference cone angle.

The force change curve of the bearing block with the torque patients need is shown in Fig. (6). It can be seen that forces increase in proportion to the torque. The larger the torque the patients need, the heavier the strength theAFO bears. So, structure optimization is needed to guarantee the AFO's intensity.

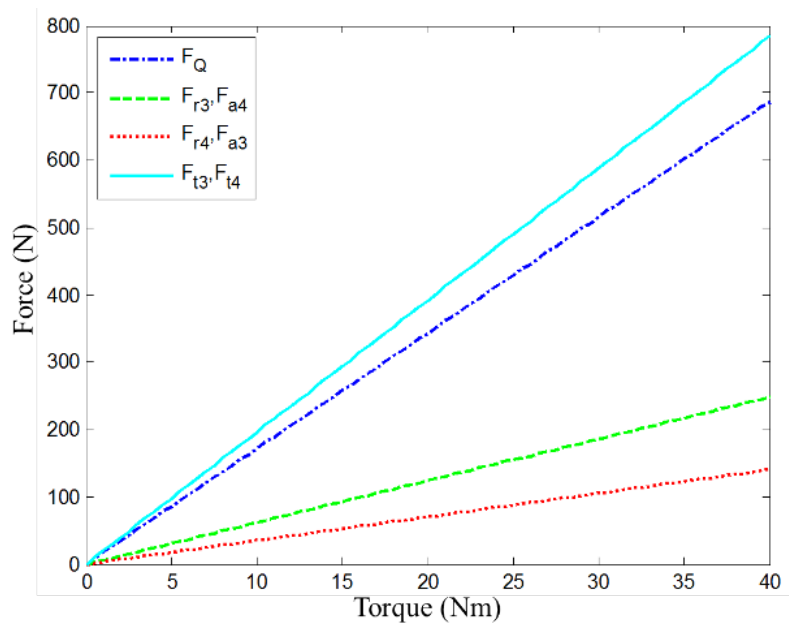

Fig. (6). Force change curve of bearing block with the torque patient needs.

All forces are transmitted to the bearing block as shown in Fig. (5c) and then they are put on the installation parts of the bearing block in SolidWorks simulation. The analysis of primal structure and modified structure is shown in Fig. (7). From the finite element analysis in Fig. (7a), there is higher material stress near the bolt hole and the maximum stress exceeds the yield strength by $325 \mathrm{MPa}$, so the basic method for strengthening rib and increasing the material thickness is adopted to increase bearing block's strength. In Fig. (7b), the results show that the method is effective and the strength conforms to the design requirements.

\subsection{Control System}

The control system is composed of software and hardware platform: the software platform utilized IAR
EWARM development environment and control program; the hardware platform mainly consists of mechanical structure of the P-AFO, control board, 24V lithium battery as the power supply, brushless DC servomotor, encoder, ground reaction force sensors (Tekscan's FlexiForce Sensors), motor driver and some auxiliary circuit. The experiment platform schematic diagram is shown in Fig. (8). To ensure the motor motion curve as close as possible to human ankle motion curve, the proportion-integration-differentiation (PID) closed-loop control is used in the P-AFO system. The main task of the control system is to realize the motor position and speed control. Control system directly affects the P-AFO's motion performance. LM3S2965 microcontroller based on ARM Cortex-M3 is the core processor of the P-AFO. Serial port is used for carrying out wireless communication between PC and the controller.

(a)

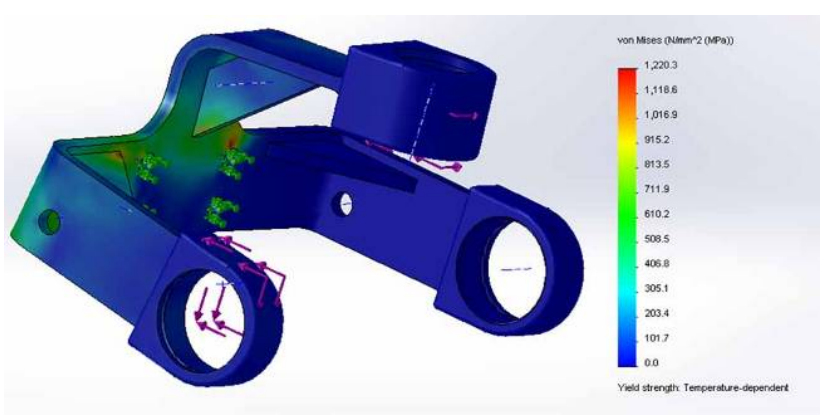

(b)

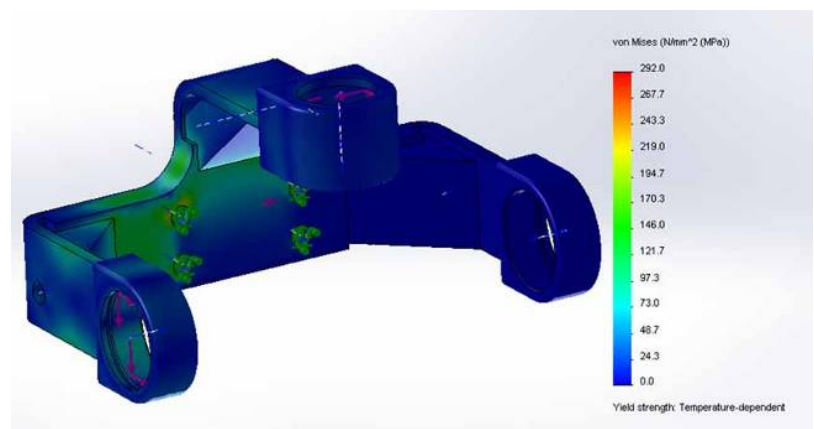

Fig. (7). Finite element analysis of bearing block.

\subsection{Complete Structure}

After a series of structure design and optimization, and assembling every transmission module and wearing parts, the SolidWorks model (Fig. 9a) and physical prototype (Fig.

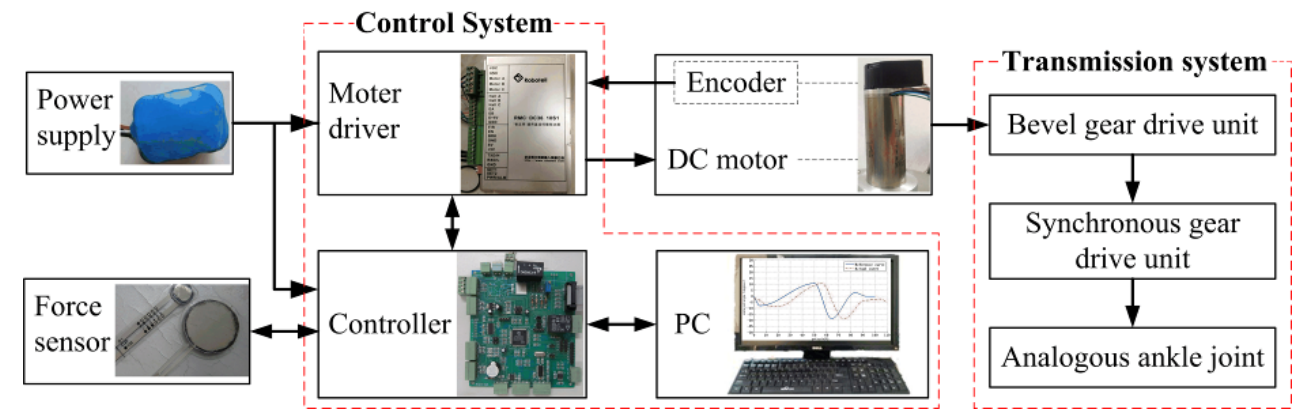

Fig. (8). Experiment platform schematic diagram. 
9b) of P-AFO are presented. Structure sizes are displayed in Table 1.

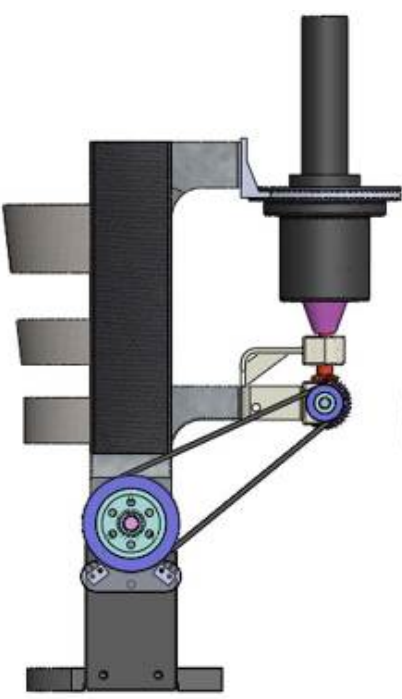

(a)

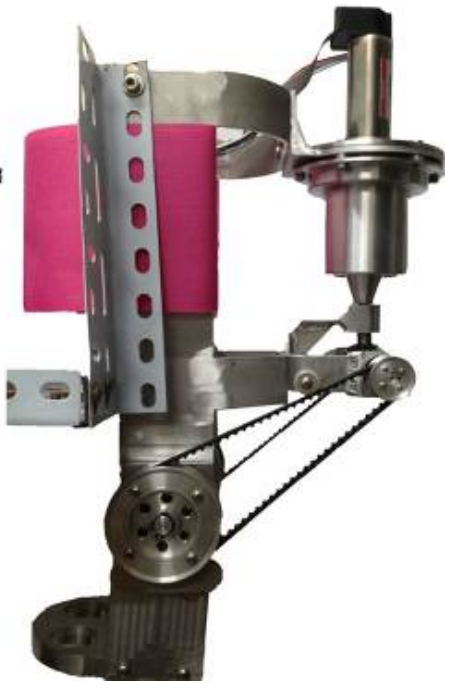

(b)
Fig. (9). Complete structure of P-AFO.

Table 1. Structure sizes.

\begin{tabular}{|c|c|c|c|}
\hline Structure Name & Length (mm) & Width (mm) & Height (mm) \\
\hline \hline Overall structure & 245 & 172 & 443 \\
\hline Lower leg part & 132 & 98.5 & 272 \\
\hline Foot part & 128 & 100 & 140 \\
\hline Bearing & 113.4 & 68.5 & Diameter (mm) \\
\hline Structure Name & Thickness (mm) & 35 \\
\hline \hline Big bevel gear & 10.5 & 19 \\
\hline Small bevel gear & 5.5 & 198 \\
\hline Big pulleys & 13.5 & Maximum Diameter (mm) \\
\hline Small pulleys & 13.5 & 100 \\
\hline Structure Name & Length (mm) & \multicolumn{2}{|c|}{5} \\
\hline \hline Reductor units & 190 & \multicolumn{2}{|c|}{19} \\
\hline
\end{tabular}

In order to validate that the mechanical structure of the PAFO can satisfy the lower leg wearing requirement in different sizes, two healthy adult subjects are chosen for tests as shown in Table 2. An adult male subject with the complete P-AFO system is shown in Fig. (10). To guarantee the portability during user's walking, the control board, motor driver and power supply are strapped to the user's waist.

The subjects' feedback illustrates that the ankle-foot orthosis is convenient and comfortable to wear and the participants can complete normal knee flexion. The front end of AFO's sole is located at participant's mid-foot position, so they can also perform toe flexion normally. In addition, there is enough space between participant's ankle-foot part and harmonic drive reducer, gear drive and timing-belt drive units to ensure a safe distance from moving parts. The design size of lower leg and foot wearing parts are rational.

Table 2. Subjects' condition.

\begin{tabular}{|c|c|c|}
\hline Adult Subject & Female & Male \\
\hline \hline Age & 26 & 25 \\
\hline Height & $165 \mathrm{~cm}$ & $178 \mathrm{~cm}$ \\
\hline Weight & $48 \mathrm{~kg}$ & $70 \mathrm{~kg}$ \\
\hline Affect side & Left/right & Left/right \\
\hline Lower leg girth & $33 \mathrm{~cm}$ & $40 \mathrm{~cm}$ \\
\hline Foot length & $32 \mathrm{~cm}$ & $26 \mathrm{~cm}$ \\
\hline $\begin{array}{c}\text { Lower leg length } \\
\text { (the length between ankle to knee) }\end{array}$ & $43 \mathrm{~cm}$ & $46 \mathrm{~cm}$ \\
\hline $\begin{array}{c}\text { Distance between AFO } \\
\text { motor and subject's knee }\end{array}$ & $9 \mathrm{~cm}$ & $12 \mathrm{~cm}$ \\
\hline $\begin{array}{c}\text { Distance between top of AFO } \\
\text { mechanism and subject's knee }\end{array}$ & $16 \mathrm{~cm}$ & $19 \mathrm{~cm}$ \\
\hline \multicolumn{2}{|c}{} \\
\hline
\end{tabular}

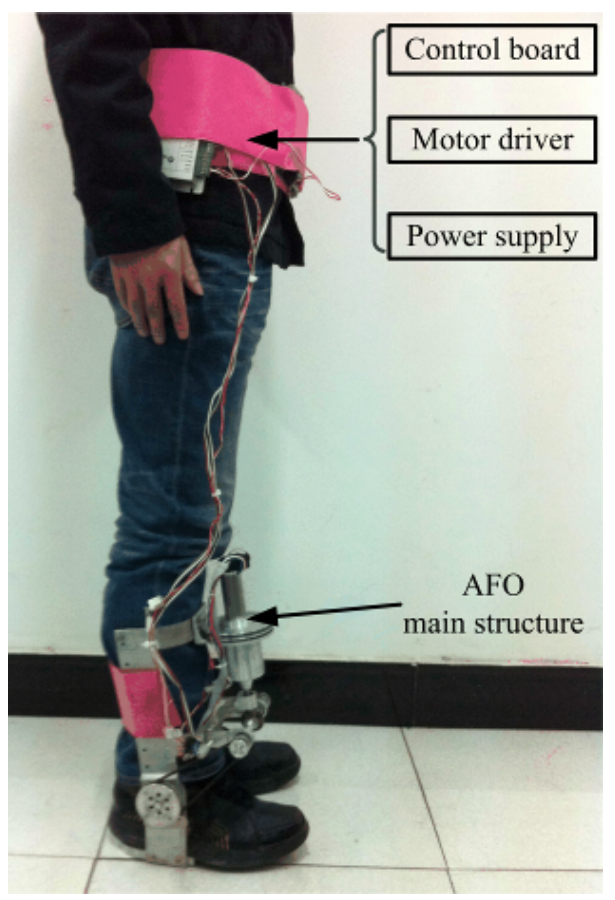

Fig. (10). Subject wearing P-AFO in standing position.

\section{EXPERIMENTS}

Aiming at patients' different requirements in different rehabilitation stages, the P-AFO has two training modes: fixed training and free training modes. In the early rehabilitation stage, because patients with serious ankle injury cannot stand normally, they can take rehabilitation training in the fixed training mode with fixed cycle and constant speed in the bed or chair. In the late rehabilitation stage, when the ankle recovers to some extent, the patient can walk and adopt free training mode. The P-AFO provides assisted torque and controls motor motion based on different stage of gait cycle. 
Experiments have been carried out to test the motion performance of AFO imitating ankle's dorsi-plantar flexion to verify whether the mechanical drive system can transfer motion and power effectively under the control system, and to record the ankle joint dorsi-plantar flexion angle, angular velocity and motion period. Each performance index of the AFO is evaluated comprehensively to find practical problems during the experimental process and then improve the system further. The main experiment contents are as follows:

- Motion performance in the fixed training mode and in subjects wearing modeis tested;

- Transmission performance of harmonic drive reducer module, bevel gear drive and synchronous belt drive module, and mechanical structure strength of the AFO with the load is tested;

- $\quad$ Position control properties of the brushless DC motor under PID control are properties;

The experiment tests the systematic performance in the following modes: the no-load mode is to test the AFO's plantar-dorsiflexion motion performance without human intervention; the walking-aid mode is to test the motion performance when subject is wearing it.

\subsection{No-Load Mode}

Under the no-load condition, the P-AFO is fixed on a bracket. The motor is controlled according to the motion curve of human gait, and an encoder records plantar flexion angle, dorsiflexion angle, angular speed and motion period of the ankle joint.

The motion cycle of the P-AFO can be adjusted according to the patients' recovery progress and the actual application.

Regarding weaker walking ability of the patients with ankle injuries, many experiments with different cycles which are longer than when conducted on normal persons are carried out to verify the control performance. The gait period 6s which is five times as much as that of normal person is chosen randomly here as an example to demonstrate the experiment results.

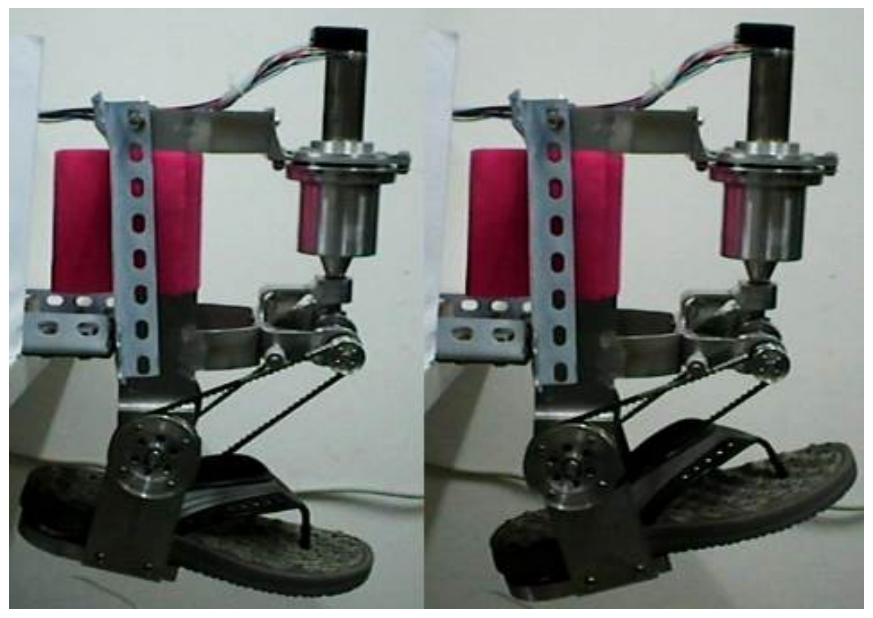

(a)

(b)

Fig. (11). Motion experiment in no-load mode.
As shown in Fig. (11), the maximum plantar flexion (Fig. 11a) and dorsiflexion (Fig. 11b) angle of the powered AFO are set at $12^{\circ}$ and $18^{\circ}$ respectively, and the motion range reaches $30^{\circ}$. The maximum angle can be changed by the program based on the specific patient's rehabilitation requirements to achieve the aim of wide application.

Ten complete cycles of motion are performed by the controller. The run-time of every cycle and the results are shown in Table 3. The average time of a complete gait (from the maximum plantar flexion position to the same position again) is $T=\left(\sum_{i=1}^{10} T_{i}\right) / 10=6.34 \mathrm{~s}$.

Table 3. Actual gait period.

\begin{tabular}{|c|c|c|c|}
\hline Sequence & Period(s) & Sequence & Period(s) \\
\hline \hline 1 & 6.23 & 6 & 6.54 \\
\hline 2 & 6.42 & 7 & 6.46 \\
\hline 3 & 6.18 & 8 & 6.30 \\
\hline 4 & 6.26 & 9 & 6.28 \\
\hline 5 & 6.33 & 10 & 6.39 \\
\hline
\end{tabular}

To assess the control performance of the powered AFO, the reference curve and actual motion curve motor imitating the ankle joint plantar-dorsiflexion are shown in the same plot in Fig. (12). Compared with the reference curve, the actual motion has a certain time delay resulting from the bandwidth limits of the motor driver and the influences of frictions.

Although the gait period of actual motion is $6 \mathrm{~s}$, the shapes of the two curves are nearly identical and the actual motion has typical features of a gait cycle, so the AFO can provide rehabilitation training for patients with ankle injury. The experimental results have verified the effectiveness of motor position control.

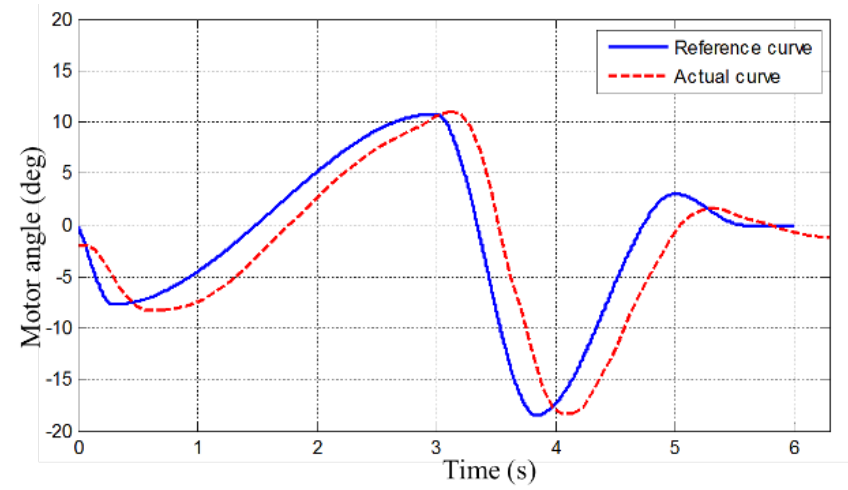

Fig. (12). Reference curve and actual curve of plantar-dorsiflexion.

\subsection{Walking-Aid Mode}

As shown in Fig. (13), an adult male participant is wearing the P-AFO to walk under its assistance for testing the systematic performance. The pictures of three gait cycles are captured from the video: the first period is $5 \mathrm{~s}-10 \mathrm{~s}$, the second period is $11 \mathrm{~s}-16 \mathrm{~s}$, and the third period is $17 \mathrm{~s}-22 \mathrm{~s}$. 

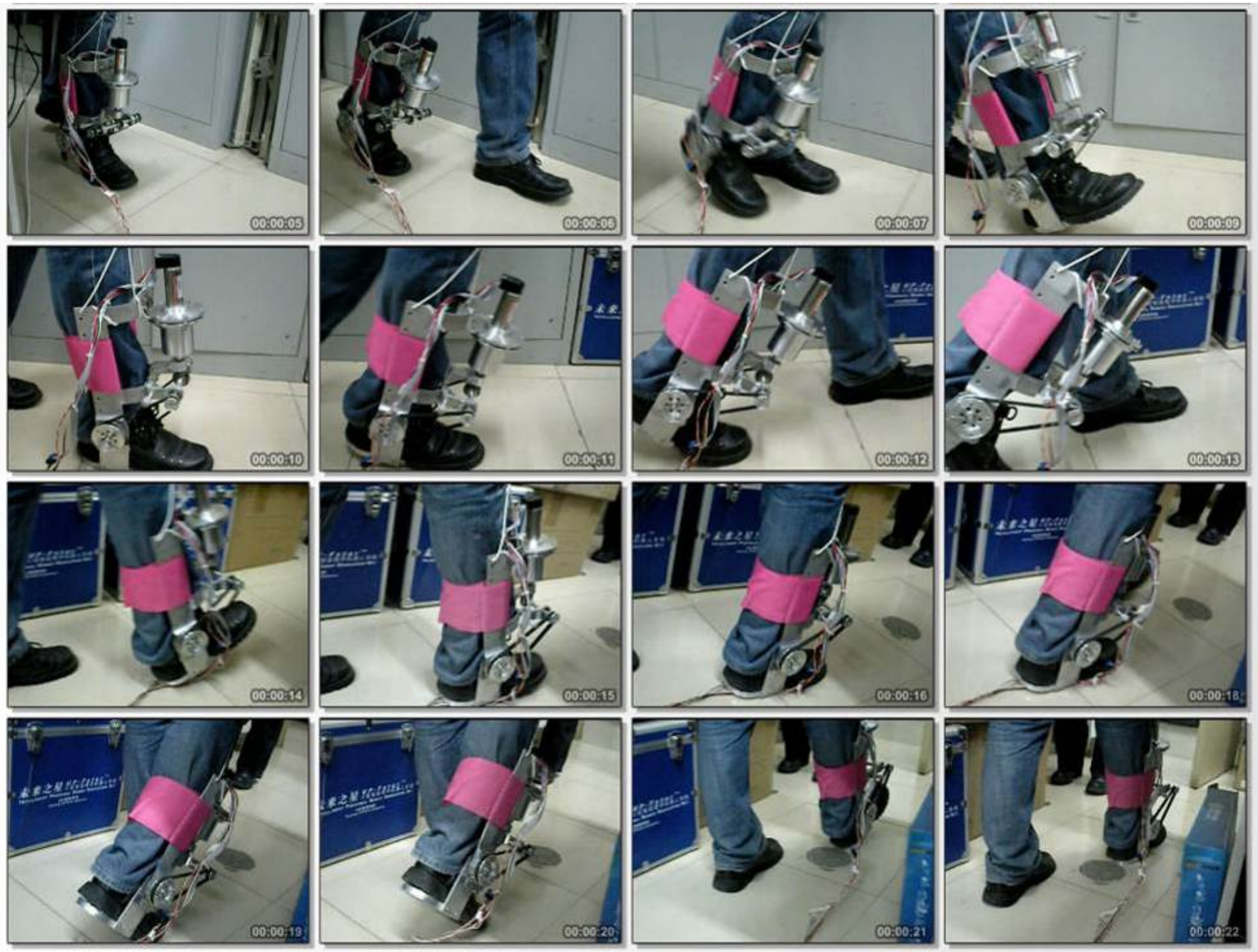

Fig. (13). Motion experiment in walking-aid mode.

Although healthy people have more active movement than ankle injury patients, the subject does not feel uncomfortable with the P-AFO. The experimental results show that transmission system is stable and reliable.

\section{CONCLUSION}

This paper proposed a novel P-AFO with motor-drive mode for ankle rehabilitation by providing plantardorsiflexion assistance to ankle patients. A dynamics model was established and a motion gait curve was planned by researching the human motion. Mechanism design affects AFO's function realization directly, so, after studying on the actuator, the way of wearing and driving mode of AFO, a new mechanical structure was presented. To improve control accuracy and make the motor motion curve as closely as possible to human gait curve, the PID closed-loop control was used in the motor position and speed control. At last, wearing and motion experiments were carried out which validated the rationality of the mechanics structure and control system, and demonstrated satisfactory results.

The P-AFO has the following characteristics and functions:
- Its shape and size can satisfy the requirements of human ankle physiological structure.

- The ankle joint's movement range can meet the demands of the angle variation of ankle plantar flexion and dorsiflexion during normal walking.

- The transmission system composed of harmonic drive, bevel gear and synchronous belt units ensures the structure compactness and strength.

- $\quad$ The P-AFO with motor-drive mode is so portable, lightweight and comfortable that patients can use it during walking to overcome the weakness of the common ankle rehabilitation devices limiting the patients within fixed places.

There are, however, certain deficiencies remaining which need to be improved and enhanced. For example, the motor controlling curve was planned based on healthy people first and then adjusted by patients' feeling. At present, efforts are being made to control the patient's injured foot according to the plantar pressure sensors data collected from the patient's healthy foot. Although the preliminary experiment results show much promise, more foot-disorder patients are needed to carry out clinical trials. 
The developing tendency of the ankle joint rehabilitation devices is as follows:

- Gait parameters are used to assess the performance and clinical effect of ankle rehabilitation devices;

- $\quad$ Ankle rehabilitation devices have the characteristics of light weight, comfort, safety and efficiency;

- The devices can provide torque for patients in walking-aid mode;

- $\quad$ There are intelligent control systems in AFO, such as emergency braking system, dynamic information feedback system and compliance control system.

\section{CONFLICT OF INTEREST}

The authors confirm that this article content has no conflict of interest.

\section{ACKNOWLEDGEMENTS}

This work was supported by Capital Medical Development Scientific Research Fund (No. 20092098) and PhD Program Foundation of Ministry of Education of China (No.20121101110011).

\section{REFERENCES}

[1] Global Stroke Leader Calls for Urgency on World Stroke Day [Internet], World Stroke Organization, updated 2013 October 29, cited 2013 November 29, [Available from: http://www.worldstroke.org/]

[2] M. Zhang, T. C. Davies and S. Xie, "Effectiveness of robot-assisted therapy on ankle rehabilitation- a systematic review," J. Neuroeng. Rehabil., vol.10, p. 30, 2013.

[3] E.A. Middleton, G.R.B. Hurley, and J.S. McIlwain, "The role of rigid and hinged polypropylene ankle-foot-orthoses in the management of cerebral palsy: a case study," Prosthet. Orthot. Int., vol. 12 , no. 3 , pp. $129-135,1988$.

[4] H. Herr, and R. Kornbluh, "New horizons for orthotic and prosthetic technology: artificial muscle for ambulation," In: Proceeding SPIE 5385, Smart Structures and Materials 2004: Electroactive Polymer Actuators and Devices, San Diego, CA, USA, 2004. pp. 1-9.

[5] J. Furusho, T. Kikuchi, M. Tokuda, T. Kakehashi, K. Ikeda, S. Morimoto, Y. Hashimoto, H. Tomiyama, A. Nakagawa and Y. Akazawa, "Development of shear type compact MR brake for the intelligent ankle-foot orthosis and its control," In: Proceeding 2007 IEEE Int. Conf. on Rehabilitation Robotics, Noordwijk, Netherlands, 2007, pp. 89-94.

[6] D. A. Winter, and S. E. Sienko, "Biomechanics of below-knee amputee gait," J. Biomech, vol. 21, pp.361-367, 1988.

[7] J. J. Eng, and D. A. Winter, "Kinetic analysis of the lower limbs during walking: what information can be gained from a threedimensional model," J. Biomech., vol. 28, no. 6, pp. 753-758, 1995.
[8] B. Brackx, M. Van Damme, A. Matthys, B. Vanderborght and D. Lefeber, "Passive ankle-Foot prosthesis prototype with extended push-off," Int. J. Adv. Robot. Syst., vol. 10, pp. 101, 2013.

[9] D. Sanz-Merodio, M. Cestari, J. C. Arevalo and E. Garcia, "Control motion approach of a lower limb orthosis to reduce energy consumption," Int. J. Adv. Robot. Syst., vol. 9, p. 232, 2012.

[10] R. Chin, E. T. Hsiao-Wecksler, E. Loth, G. Kogler, S. D. Manwaring, S. N. Tyson, K. A. Shorter and J. N. Gilmer, "A pneumatic power harvesting ankle-foot orthosis to prevent footdrop," J. Neuroeng. Rehabil., vol. 6, p. 19, 2009.

[11] K. A. Shorter, G. F. Kogler, E. Loth, W. K. Durfee, and E. T. Hsiao-Wecksler, "A portable powered ankle-foot orthosis for rehabilitation," J. Rehabil. Res. Dev., vol. 48, no. 4, pp. 459-472, 2011.

[12] K. E. Gordon, G. S Sawicki, and D. P. Ferris, "Mechanical performance of artificial pneumatic muscles to power an ankle-foot orthosis," J. Biomech., vol. 39, pp. 1832-1841, 2006.

[13] D. P. Ferris, K. E. Gordon, G. S. Sawicki, and A. Peethambaran, "An improved powered ankle-foot orthosis using proportional myoelectric control," Gait Posture, vol. 23, no. 4, pp. 425-428, 2006.

[14] S. M. Cain, K. E. Gordon, and D. P. Ferris, "Locomotor adaptation to a powered ankle-foot orthosis depends on control method," $J$. Neuroeng. Rehabil., vol. 4, p. 48, 2007.

[15] D. P. Ferris, J. M. Czerniecki, and B. Hannaford, "An ankle-foot orthosis powered by artificial pneumatic muscles," J. Appl. Biomech., vol. 21, no. 2, pp. 189-197, 2005.

[16] M. Noel, B. Cantin, S. Lambert, C. M. Gosselin and L. J. Bouyer, "An electrohydraulic actuated ankle foot orthosis to generate force fields and to test proprioceptive reflexes during human walking," IEEE Trans. Neural Syst. Rehabil. Eng., vol. 16, no. 4, pp. 390399, 2008.

[17] J. A. Blaya, and H. Herr, "Adaptive control of a variableimpedance ankle-foot orthosis to assist drop-foot gait," IEEE Trans. Neural Syst. Rehabil. Eng., vol. 12, no. 1, pp. 24-30, 2004.

[18] J. Ward, T. Sugar, J. Standeven, and J. R. Engsberg, "Stroke survivors' gait adaptations to a powered ankle-foot orthosis," Adv. Robotics, vol. 25, no. 15, pp. 1879-1901, 2011.

[19] S. Au, M. Berniker, and H. Herr, "Powered ankle-foot prosthesis to assist level-ground and stair-descent gaits," Neural Network, vol. 21, no. 4, pp. 654-666, 2008.

[20] J. Enderle, S. Blanchard, and J. Bronzino, "Biomechanics," In: Introduction to Biomedical Engineering, $2^{\text {nd }}$ ed., J. Bronzino, Ed., San Diego: Elsevier Academic Press, 2005, pp. 127-210.

[21] Y. Bai, F. Li, J. Zhao, J. Li, F. Jin and X. Gao, "A powered ankleFoot orthoses for ankle rehabilitation," In: Proceeding 2012 IEEE International Conference on Automation and Logistics, Zhengzhou, China, pp. 288-293, 2012.

[22] J. Rose and J. G. Gamble. Human walking, $3^{\text {rd }}$ ed., Lippincott Williams \& Wilkins, Philadelphia, 2006.

[23] W. K. Lam, J. C. Y. Leong, Y. H. Li, Y. Huand, and W. W. Lu, "Biomechanical and electromyographic evaluation of ankle foot orthosis and dynamic ankle foot orthosis in spastic cerebral palsy," Gait Posture, vol. 22, no. 3, pp. 189-197, 2005.

[24] X. Zhu, "Synchronous Belt Drive," In: Handbook of mechanical transmission design, Beijing: Publishing House of Electronics Industry, pp. 1012-1045, 2007.

[25] H. Rong, T. Fu, and M. Yang, "Gear Drive," In; Introduction to mechanical design, $2^{\text {nd }}$ ed., Beijing Institute of Technology Press, Beijing, 2006, pp. 76-118. 